\title{
Disparities in Oral Healthcare Access Due to the COVID-19 Pandemic
}

\author{
Fnu Namrata1, Zvi G. Loewy ${ }^{2,3 *}$ \\ ${ }^{1}$ Westchester Medical Center, Valhalla, USA \\ ${ }^{2}$ Touro College of Pharmacy, New York, USA \\ ${ }^{3}$ School of Medicine, New York Medical College, Valhalla, USA \\ Email: fnamrata@nymc.edu, *zvi.loewy@touro.edu
}

How to cite this paper: Namrata, F. and Loewy, Z.G. (2021) Disparities in Oral Healthcare Access Due to the COVID-19 Pandemic. Open Journal of Stomatology, 11, 437442.

https://doi.org/10.4236/ojst.2021.1111038

Received: September 21, 2021

Accepted: November 1, 2021

Published: November 4, 2021

Copyright $\odot 2021$ by author(s) and Scientific Research Publishing Inc. This work is licensed under the Creative Commons Attribution International License (CC BY 4.0).

http://creativecommons.org/licenses/by/4.0/

\begin{abstract}
The U.S. has experienced very high numbers of positive COVID-19 cases, along with high unemployment rates during the COVID-19 pandemic. Similarly, on a global level, the pandemic has resulted in an increase in the unemployment rate, loss of insurance and an overall adverse social impact. In the social sector, there has been a huge gap in supply and demand; this has been especially apparent in the healthcare industry. The COVID-19 pandemic has had a significant negative impact on high-contact industries, including dentistry. Causative factors have included social distancing, shortage of healthcare professionals and loss of insurance due to unemployment. The result has been a void in preventative oral health services. Limited information on the disproportionate access to oral healthcare, due to the increase in unemployment and corresponding loss of insurance during the COVID-19 pandemic is available. This study was designed to investigate the disparities in access to oral healthcare amidst the global economic crisis and rise in unemployment.
\end{abstract}

\section{Keywords}

Economic Impact, Health Insurance, Inequalities

\section{Introduction}

Reported in Wuhan China in December 2019, COVID-19 is an ongoing global pandemic, caused by the severe acute respiratory syndrome coronavirus 2 (SARSCoV-2). The World Health Organization (WHO) declared a Public Health Emergency of International Concern in January 2020. The consequences of the pandemic are beyond the disease itself; it will have a long-lasting impact on the global economy. According to the International Labor Organization, in April 2020 more 
than 20 million Americans filed for unemployment insurance, and the national unemployment rate was reported as $14.7 \%$, the highest monthly rate since record keeping began in 1948. In health care, spending dropped 18 percent and tens of thousands of health care workers lost their jobs. Overall, a broad uncertainty of the world economic system exists amidst the recovery and containment [1] [2].

The COVID-19 pandemic has also raised concerns over racial and geographic discrimination, health equity, and disparities between public health imperatives and individual rights. In the U.S., there exists a huge disparity in healthcare affordability and accessibility. According to The Centers for Disease Control and Prevention (CDC), non-Hispanic blacks, Hispanics, and American Indians and Alaska Natives have been affected by the oral health disparities at a higher rate than other populations [3]. Evidence suggests that the COVID-19 pandemic exacerbated the disproportionate access to oral health care in these populations.

In this study, we focus on the disparities in healthcare access, especially in the field of oral health during the COVID-19 pandemic. A review of the literature was performed using Medline/PubMed/Embase database resources for English language papers to identify appropriate articles that addressed the objectives of this study. A variety of keywords were employed in keyword/title/abstract searches that included: oral health disparities, healthcare affordability, healthcare accessibility, pandemic, COVID-19, SARS-CoV-2. Publications were reviewed independently by two investigators. The investigators extracted the data and inspected each reference identified by the search. In cases where the same studies were reported in more than one publication, the study's results were accounted for only once. Limits to the search strategy were English-language articles. The electronic search was followed by extensive hand-searching using reference lists from the identified articles.

Study objectives include:

1) To obtain insight into the economic impact of the pandemic resulting from unemployment and loss of Employer-Sponsored health Insurance (ESI);

2) To evaluate the adverse impact of the pandemic on oral healthcare access;

3) To suggest an approach to resolve the disparities.

\section{Relationship of COVID-19 and Health Insurance}

Even before the pandemic, the U.S. was facing the challenge of a persistent gap in access to high quality healthcare, and its affordability. There is a significant gap with insurance coverage in the U.S. population. According to the U.S. Census Bureau (2018 data), non-Hispanic white people had an uninsured rate of 5.4 percent as compared to 6.8 percent for Asian people, 9.7 percent for Black people, and 17.8 percent for Hispanic people of any race. 13.8 percent of individuals in households making less than $\$ 25,000$ a year did not have health insurance as compared to 3.2 percent of households with an income of $\$ 150,000$ a year or greater [4]. In the U.S., ESI which is employer based, is the largest source of health coverage, and is most vulnerable in these times of the pandemic when many people 
have lost their jobs [4].

Amidst, the global pandemic, it is estimated that 17.7 million people could lose access to their ESI if the unemployment rate rises to 15 percent in the U.S. It is estimated that $71 \%$ would regain insurance coverage through Medicaid, the Affordable Care Act (ACA) individual market, or other private coverage. That said, this will still leave over 5 million people uninsured in the U.S. [5]. Disparity in ESI coverage varies considerably by race, gender, and location, along with the high unemployment rate. Not having health insurance means, uninsured individuals will not seek care in the early stages of disease and elect to wait until their condition declines and requires hospitalization. Health insurance plays a vital role in the management of chronic disease that could deteriorate without continuity in access to appropriate care [6].

\section{Impact of COVID-19 on Access to Oral Healthcare}

Historically, populations with poor oral health access in the U.S., included the uninsured, low-income families, immigrants, members of the racial/ethnic minorities, and rural populations with suboptimal access to quality oral healthcare [7]. COVID-19 significantly impacted the dental health services, with practice closures and reduced working hours; with the exception of urgent and emergency services. Those populations at high risks for COVID-19 disproportionately experienced limited access to oral health, while the patients who manifested disease symptoms were advised to avoid non-emergency oral healthcare [8]. The growing evidence from the literature shows that healthcare providers were advised to consider delaying dental care services of symptomatic patients until the full recovery was achieved. According to Brian and Weintraub [4], over forty-nine million people in the United States live in areas designated by the "Health Resources and Services Administration", as oral health "Professional Shortage Areas." The data suggests that emergency departments received limited visits for oral health services in the peak of COVID-19 [9]. For children during the pandemic, school closures resulted in suspension of school-based dental programs such as dental caries prevention, exacerbating the inequality [10]. Statistics show a significant inequality in the provision of sealant services between the low-income and economically average families. According to Brian and Weintraub [4], children from low-income families are less likely to receive sealant services (39\%) as compared to those from the higher-income families (46\%).

Patients with more serious dental health problems faced a significant challenge during the COVID-19 pandemic. Multiple uncertainties, including the potential risks from dental procedures' infectious aerosols, airborne virus transmission, and the shortage of Personal Protective Equipment (PPE) resulted in the worldwide authorities instituting guidance that significantly limited dental services [10].

Access inequalities are particularly acute for the minority and vulnerable populations, low-income families are less likely to have dental insurance [9]. Evidence shows that many states eliminated Medicaid for oral health coverage over 
the last decades due to fiscal challenges stemming from the current $10 \%$ decline in dental care utilization among low-income adults [11]. Additionally, the at-risk populations with dental coverage under Medicare, rarely find Medicaid contracted dental providers because the federal dentists accepting Medicaid accounts for only $20 \%$. Medicaid programs were already subject to high administrative requirements and limited participants in dental healthcare even before the COVID-19 pandemic [8]. Populations that rely on Medicaid for oral health benefits experienced significant challenges, as their access to dental healthcare services was limited, or restricted, by reducing essential coverage [10].

\section{How COVID-19 Placed the Populations with Oral Health Disparities at Elevated Risks}

The populations with oral health diseases are similar to the populations developing chronic health conditions. The typically cited risk factors for these conditions includes tobacco and alcohol use, poor diet, behavioral health issues, substance abuse, poverty, stress, and domestic violence [4]. Many of these factors gained prevalence during the COVID-19 pandemic, and along with other social determinants of health, it exacerbated oral health outcomes. Evidence from extensive studies show that populations positive for COVID-19 included minority groups, the low socioeconomic groups, low-literacy individuals, older adults, the uninsured populations, and those from rural areas [12]. These populations also manifested increased risks for dental diseases and associated systemic health problems. Overwhelming evidence shows that minority populations were at a high risk for adverse dental health outcomes during the COVID-19 pandemic [8]. Of concern, the low socioeconomic groups, low-literacy groups, uninsured and those from rural areas have had greater and unprecedented incidences of COVID-19-related deaths and infections [13].

\section{Approach to Resolve Disparities: Lessons from COVID-19}

The literature informs several lessons drawn from the COVID-19 pandemic and its implication to dentistry. Studies confirm that: 1) dental health must be an integral component of the essential medical system [4];2) It is imperative to reduce dental health disparities; 3) Responsibility lies with the stakeholders, including dental associations, governments, the insurance industries, and employees, to increase the availability, affordability, and accessibility for all constituents. The loss of oral health insurance coverage and the service limitation that was more prevalent during the pandemic predisposed millions of people to adverse outcomes due to health disparities [4].

\section{Approach to Resolve Disparities: Integrating the Conceptual Framework}

The pandemic's indirect impacts are extensive, pervasive, and complex; and they arise from restricted access to dental healthcare [14]. Therefore, the ramifica- 
tions can be underpinned using Andersen's 2014 theory of health service use [14]. This comprehensive model explains the interaction between people, the external environment, and the healthcare systems [15]. Multiple factors, including cultural, economic, social, and individual characteristics, primarily predispose people to accessing healthcare [14]. The model also posits that the enabling factors, including health services organization, governance, and financing, may decrease or increase access to healthcare [14]. Patients' behavioral factors, including professional and personal, also influence access to healthcare services [15]. The model is pertinent because it links patient satisfaction as desired outcomes to health; this theory is therefore relevant to understanding access to dental healthcare [14].

\section{Conclusion}

The COVID-19 pandemic has affected many people worldwide. Significantly, it has had a disproportionate impact on the disadvantaged population in the U.S. The disparity in healthcare access, plagued by the pandemic, is unjust. Health is a fundamental human right, and so too is dental health. In most societies and healthcare systems, health services are denoted as public goods, implying a significant share of governmental or public responsibility to provide healthcare services and ensure equitable dental health accessibility to the entire population [16]. Evidence shows that when dental healthcare and essential health are not part of the primary healthcare or public healthcare systems, appropriate financial support should be provided [11]. It is crucial to consider disparities in oral health status at the time of the COVID-19 pandemic. Initiators of healthcare policies, and policy stakeholders must work to address this crisis, ensuring equitable access to oral healthcare to improve the overall quality of population health and quality of life.

\section{Conflicts of Interest}

The authors declare no conflicts of interest regarding the publication of this paper.

\section{References}

[1] Wikimedia Foundation (2021) Economic Impact of the COVID-19 Pandemic. Wikipedia.

https://en.wikipedia.org/wiki/Economic impact of the COVID-19 pandemic\#Un employment

[2] Holzer, H.J. (2020) COVID Outcomes Update: Health and Employment Impacts in the US Compared to Other Countries.

[3] Berchick, E.R., Barnett, J.C. and Upton, R.D. (2019) Health Insurance Coverage in the United States: 2018. Current Population Reports, United States Census Bureau, Suitland.

[4] Brian, Z. and Weintraub, J.A. (2020) Oral Health and COVID-19: Increasing the Need for Prevention and Access. Preventing Chronic Disease, 17, Article ID: 200266. https://doi.org/10.5888/pcd17.200266 
[5] Gangopadhyaya, A. and Garrett, A.B. (2020) Unemployment, Health Insurance, and the COVID-19 Recession. https://doi.org/10.2139/ssrn.3568489

[6] Cole, B. (2020) The Impact of the COVID-19 Pandemic on Access to Health Care. National Academy of Social Insurance, Washington DC.

https://www.nasi.org/research/the-impact-of-the-covid-19-pandemic-on-access-tohealth-care/

[7] Northridge, M.E., Kumar, A. and Kaur, R. (2020) Disparities in Access to Oral Health Care. Annual Review of Public Health, 41, 513-535. https://doi.org/10.1146/annurev-publhealth-040119-094318

[8] Wang, T.T., Mathur, M.R. and Schmidt, H. (2020) Universal Health Coverage, Oral Health, Equity, and Personal Responsibility. Bulletin of the World Health Organization, 98, 719-721. https://doi.org/10.2471/BLT.19.247288

[9] Kalash, D.A. (2020) How COVID-19 Deepens Child Oral Health Inequities. Journal of the American Dental Association, 151, 643-645.

https://doi.org/10.1016/j.adaj.2020.05.015

[10] Nasir, E.F., Elhag, A.K. and Almahdi, H.M. (2020). COVID-19 Perceptional Disparity among Dental Healthcare Personnel at King Faisal University: Applying Health Belief Model. European Journal of Dentistry, 14, S56-S62. https://doi.org/10.1055/s-0040-1716782

[11] Benzian, H., Beltrán-Aguilar, E., Mathur, M.R. and Niederman, R. (2021) Pandemic Considerations on Essential Oral Health Care. Journal of Dental Research, 100, 221-225. https://doi.org/10.1177/0022034520979830

[12] Estrich, C.G., Mikkelsen, M., Morrissey, R., Geisinger, M.L., Ioannidou, E., Vujicic, M. and Araujo, M.W. (2020). Estimating COVID-19 Prevalence and Infection Control Practices among US Dentists. The Journal of the American Dental Association, 151, 815-824. https://doi.org/10.1016/j.adaj.2020.09.005

[13] Elster, N. and Parsi, K. (2021). Oral Health Matters: The Ethics of Providing Oral Health during COVID-19. HEC Forum, 33, 157-164. https://doi.org/10.1007/s10730-020-09435-3

[14] Daly, J. and Black, E.A. (2020) The Impact of COVID-19 on Population Oral Health. Community Dental Health, 37, 236-238. https://doi.org/10.1922/cdh dec20editorialdalyblack03

[15] Tolera, H., Gebre-Egziabher, T. and Kloos, H. (2020) Using Andersen's Behavioral Model of Health Care Utilization in a Decentralized Program to Examine the Use of Antenatal Care in Rural Western Ethiopia. PLoS ONE, 15, Article ID: e0228282. https://doi.org/10.1371/journal.pone.0228282

[16] Watt, R.G. (2020) COVID-19 Is an Opportunity for Reform in Dentistry. The Lancet, 396, 462. https://doi.org/10.1016/S0140-6736(20)31529-4 